\title{
Drug resistance in Campylobacter jejuni, $C$ coli, and $C$ lari isolated from humans in North West England and Wales, 1997
}

\author{
R T Thwaites, J A Frost
}

\begin{abstract}
Aims-To test the sensitivity of strains of Campylobacter species isolated from humans in England and Wales against a range of antimicrobial agents for the purpose of monitoring therapeutic efficacy and as an epidemiological marker.

Methods-An agar dilution breakpoint technique was used to screen isolates against ampicillin, chloramphenicol, gentamicin, kanamycin, neomycin, tetracycline, nalidixic acid, ciprofloxacin, and erythromycin. Minimal inhibitory concentrations (MIC) were also determined for a sample of quinolone resistant strains.
\end{abstract}

Results-Approximately $\mathbf{5 0 \%}$ of strains tested were resistant to at least one drug. Strains which were resistant to four or more of the drugs tested were classified as multiresistant; this occurred in $11.3 \%$ of $C$ jejuni, $19.9 \%$ of $C$ coli, and $63.6 \%$ of C lari. Resistance to erythromycin occurred in $1.0 \%$ of $C$ jejuni and $12.8 \%$ of C coli. Resistance to quinolones occurred in $12 \%$ of strains, with a ciprofloxacin MIC of $>8 \mathrm{mg} / 1$ and a nalidixic acid MIC of $>256 \mathrm{mg} / 1$; a further $4 \%$ of strains had intermediate resistance with a ciprofloxacin MIC of between 0.5 and $2 \mathrm{mg} / 1$ (fully sensitive strains, $0.25 \mathrm{mg} / 1$ or less) and a nalidixic acid MIC of between 32 and $64 \mathrm{mg} / 1$ (fully sensitive strains, $8 \mathrm{mg} / 1$ or less).

Conclusions-Resistance to quinolones in campylobacters from human infection may relate to clinical overuse or use of fluoroquinolones in animal husbandry. Both veterinary and clinical use should be reconsidered and fluoroquinolone drugs used only as a treatment for serious infections requiring hospital admission. Erythromycin resistance is still rare in

Accepted for publication 28 April 1999

Table 1 Minimum inhibitory concentrations (MIC) of control strains and breakpoints used in resistance screening

\begin{tabular}{|c|c|c|c|c|c|c|c|c|c|c|c|}
\hline \multirow{2}{*}{$\begin{array}{l}C R U \\
\text { ref }\end{array}$} & \multirow{2}{*}{$\begin{array}{l}\text { NCTC } \\
\text { No }\end{array}$} & \multirow[b]{2}{*}{ Species } & \multicolumn{9}{|c|}{$M I C(m g / l)$} \\
\hline & & & $A$ & $C$ & $G$ & $K$ & $T$ & $N x$ & $C p$ & $E$ & $\mathrm{Neo}$ \\
\hline C206 & 12525 & C coli & 16 & 16 & $<2$ & 16 & $<2$ & 8 & 0.125 & $>16$ & 8 \\
\hline C207 & 12505 & $C$ jejuni & 8 & 16 & $<2$ & 8 & 4 & 8 & 0.125 & $<1$ & 4 \\
\hline C1096 & 10842 & $C$ fetus & $<2$ & 16 & $<2$ & 16 & 4 & 128 & 0.5 & $<1$ & 4 \\
\hline C1097 & 11352 & C lari & 256 & 8 & $<2$ & 64 & $<2$ & $>64$ & 4 & $<1$ & 8 \\
\hline C1098 & 9001 & E coli & 8 & 32 & $<2$ & 16 & 8 & 8 & 0.06 & $>16$ & 8 \\
\hline C8436 & - & $C$ jejuni & 16 & 8 & $>32$ & 64 & $>128$ & $>256$ & 16 & $<1$ & $>64$ \\
\hline
\end{tabular}

A, ampicillin; CRU, Campylobacter Reference Unit; C, chloramphenicol; Cp, ciprofloxacin; E, erythromycin; G, gentamicin; K, kanamycin; NCTC, National Collection of Type Cultures; $\mathrm{Neo}$, neomycin; $\mathrm{Nx}$, nalidixic acid; $\mathrm{T}$, tetracycline.
C jejuni but much more common in C coli.

(F Clin Pathol 1999;52:812-814)

Keywords: Campylobacter spp; drug resistance

The significance of acute enteric disease caused by Campylobacter species was only appreciated in the 1970 s, with the advent of improved isolation methods. ${ }^{1}$ Campylobacter spp have been the commonest reported cause of acute bacterial enteritis in England and Wales since 1981, as measured by reports to the Public Health Laboratory Service (PHLS) Communicable Disease Surveillance Centre (CDSC), and there were over 50000 cases reported in $1997 .^{2}$ Several studies have indicated that more than $90 \%$ of infections are caused by to $C$ jejuni, with $C$ coli representing most of the remainder. $C$ lari comprises $<1 \%$ of the strains isolated, with other species, such as $C$ upsaliensis and $C$ fetus, only occasionally seen in clinical isolates. ${ }^{3}$

Although antibiotic treatment is not advised in the majority of cases of diarrhoeal disease caused by Campylobacter spp, in severe or prolonged illness the use of erythromycin or ciprofloxacin is recommended. ${ }^{4}$ Erythromycin resistance in clinical isolates of $C$ jejuni is still believed to be rare, although it has been more commonly reported in $C$ coli strains. ${ }^{5}$ Reina et al suggested that there was also a significant increase in erythromycin resistance in quinolone resistant strains, ${ }^{6}$ although Piddock $^{7}$ refuted this link. Bowler and colleagues ${ }^{8}$ did not detect ciprofloxacin resistant campylobacters in Oxford, UK, between 1988 and 1990, but detected $7 \%$ of strains resistant in a sample of 491 isolates in Oxford in $1996 .^{9}$ McIntyre and Lyons ${ }^{10}$ detected $1.9 \%$ ciprofloxacin resistant strains in 1991 and $4.9 \%$ between January and November 1992, while Piddock ${ }^{7}$ observed a continuing rise in the 1990s and attributed this to the licensing, in November 1993, of enrofloxacin for veterinary use within the United Kingdom.

Since April 1997, campylobacters isolated from humans with diarrhoeal disease in Wales and north west England have been referred to the Laboratory of Enteric Pathogens for typing. In this paper we describe the incidence of antimicrobial resistance in these isolates.

\section{Methods}

CULTURE AND IDENTIFICATION

Isolates from humans with acute gastrointestinal infections were sent to the Laboratory of Enteric Pathogens. All isolates were 
Table 2 Incidence of resistance to drugs tested in three most common campylobacter species isolated from humans in England and Wales between April and December, 1997

\begin{tabular}{|c|c|c|c|c|c|c|c|c|c|c|c|c|c|}
\hline \multirow[b]{2}{*}{ Species } & \multirow[b]{2}{*}{ Total $\%$} & & \multicolumn{9}{|c|}{ Per cent resistant to } & \multirow[b]{2}{*}{$\% D R$} & \multirow[b]{2}{*}{$\% M R$} \\
\hline & & & $A$ & $C$ & $G$ & $K$ & $T$ & $N x$ & $C p$ & $E$ & $\mathrm{Neo}$ & & \\
\hline$C$ jejuni & 5401 & $(93 \%)$ & 34.0 & 5.6 & 0.1 & 1.1 & 30.1 & 14.8 & 10.5 & 1.0 & 0.9 & 50 & 11.3 \\
\hline C coli & 376 & $(6.5 \%)$ & 45.5 & 2.4 & 0.5 & 4.8 & 26.9 & 26.9 & 21.8 & 12.8 & 3.2 & 55.9 & 19.9 \\
\hline Clari & 25 & $(0.5 \%)$ & 36.0 & 0.0 & 0.0 & 60.0 & 12.0 & 100 & 100 & 0.0 & 4.0 & 100 & 31.8 \\
\hline \multirow[t]{2}{*}{ All } & 5802 & $(100 \%)$ & 34.0 & 5.4 & 0.1 & 1.6 & 29.6 & 16.2 & 11.7 & 1.7 & 1.1 & 50.6 & 121 \\
\hline & \multicolumn{2}{|c|}{ Breakpoint } & 8 & 8 & 4 & 16 & 8 & 16 & 1 & 4 & 8 & & \\
\hline
\end{tabular}

A, ampicillin; C, chloramphenicol; $\mathrm{Cp}$, ciprofloxacin; DR, drug resistant (resistant to one or more antimicrobial drug); $\mathrm{E}$, erythromycin; G, gentamicin; K, kanamycin; MR, multiresistant (resistant to four or more antimicrobial drug); Neo, neomycin; $\mathrm{Nx}$, nalidixic acid; $\mathrm{T}$, tetracycline.

plated onto Columbia blood agar and grown in a variable atmosphere incubator (VAIN, Don Whitley) in an atmosphere of $87 \%$ nitrogen, $5 \%$ oxygen, $3 \%$ hydrogen, and $5 \%$ carbon dioxide for 48 hours. Six discrete colonies were then picked, pooled, and plated onto a second Columbia blood agar plate. These were incubated for 24 hours in the VAIN and growth from this plate harvested to prepare cell suspensions for further tests. All strains received were identified to species level, as well as being tested for drug resistance.

TESTING FOR RESISTANCE TO ANTIMICROBIAL DRUGS

An agar incorporation method was used for drug resistance screening and determination of minimal inhibitory concentrations (MICs). ${ }^{11}$ The antimicrobials tested were incorporated into Iso-Sensitest agar, which contained 5\% laked horse blood. The breakpoints used are shown in table 1 . These breakpoints are those published by the British Society of Antimicrobial Chemotherapy (BSAC) ${ }^{12}$ except for that of erythromycin. No published guidelines exist for erythromycin testing in campylobacter. The BSAC does not recommend a concentration for any Gram negative organism. The National Committee for Clinical Laboratory Standards (NCCLS), in the USA, recommends that intermediate resistance to erythromycin for most organisms should be defined as between 1 and $4 \mathrm{mg} / 1$ and high resistance as $>8 \mathrm{mg} / \mathrm{l}^{13}$ For the purpose of this stufy a breakpoint of 4 $\mathrm{mg} / \mathrm{l}$ was accepted, to include all those strains showing resistance. This single breakpoint screening allows large numbers of strains to be examined for drug resistance in a timely manner suited to the high level of work experienced in a reference laboratory.

Trimethoprim was included in the screening, although all campylobacter strains are intrinsically resistant, as a check on identity; trimethoprim resistance was therefore not included in this analysis.

Table 3 Minimum inhibitory concentration (MIC) values for quinolone resistant strains

\begin{tabular}{lll}
\hline & \multicolumn{2}{l}{ Median value of MIC (mg/l) (range) } \\
\cline { 2 - 3 } & $N x$ & $C p$ \\
\hline $\begin{array}{c}\text { Nx sensitive / Cp } \\
\text { sensitive ( }=20) \\
\begin{array}{c}\text { Intermediate resistance } \\
(\mathrm{n}=20)\end{array}\end{array}$ & $8(4$ to 16$)$ & $0.25(0.06$ to 0.5$)$ \\
$\begin{array}{c}\text { High level resistance } \\
(\mathrm{n}=20)\end{array}$ & $32(32$ to 64$)$ & $1(0.5$ to 1$)$ \\
\hline
\end{tabular}

$\mathrm{Cp}$, ciprofloxacin; $\mathrm{Nx}$, nalidixic acid.
For drug resistance screening, a suspension equivalent to a turbidity of McFarland 1 was made in a brain-heart infusion broth. After incubation at $37^{\circ} \mathrm{C}$ for four hours, $0.5 \mathrm{ml}$ of each broth was then loaded into one well of a 36 well block for use with a Denley multipoint inoculator. Each antibiotic plate was inoculated with 30 strains and six control strains using a multipoint inoculator (table 1). For controls, National Collection of Type Cultures (NCTC) strains were used, except for C8436 which is a multiresistant strain of $C$ jejuni serotype HS50 isolated from a patient in 1997. The plates were incubated for 48 hours in the VAIN at $37^{\circ} \mathrm{C}$ and growth on the plates was scored in comparison with a non-selective control plate.

\section{Results}

The majority of isolates (93\%) were $C$ jejuni. Of the remainder, $6.5 \%$ were $C$ coli and $0.5 \%$ C lari. The incidence of drug resistance in campylobacters in these strains is shown in table 2. Strains resistant to four or more of the drugs were termed multiresistant. Drug resistance to one or more drugs was detected in over $50 \%$ of strains; multiresistance was found in $11 \%$ of $C$ jejuni strains but was more common in $C \operatorname{coli}(20 \%)$ and $C$ lari $(60 \%)$, although $C$ lari are constitutively resistant to quinolones.

In $C$ jejuni, $34 \%$ of strains tested were resistant to ampicillin, but less than $2 \%$ of strains were resistant to aminoglycosides and erythromycin. All 43 isolates of $C$ jejuni resistant to kanamycin were also resistant to tetracyclines. Resistance to kanamycin was common in those strains of $C$ lari tested ( $60 \%$ ), but tetracycline resistance was less common (12\%). C coli, which make up $6.5 \%$ of the total strains tested, were generally more resistant. Erythromycin resistance was seen in only $1.0 \%$ of $C$ jejuni, but in $12.8 \%$ of $C$ coli strains. All 25 strains of $C$ lari tested were sensitive to erythromycin.

$C$ jejuni and $C$ coli can be divided into three groups with respect to quinolone resistance, as is shown by full MICs done on a subset of 60 isolates (table 3). This set was selected as a sample of 20 strains each of those resistant to ciprofloxacin and nalidixic acid, nalidixic acid only, or sensitive to both, by primary breakpoint analysis. Fully sensitive strains $(88.3 \%)$ were sensitive to nalidixic acid at $16 \mathrm{mg} / \mathrm{l}$ and ciprofloxacin at $1 \mathrm{mg} / \mathrm{l}$. An intermediate group comprising $4.3 \%$ of strains appeared as nalidixic acid resistant and ciprofloxacin sensitive in the routine screen (table 2). These had an MIC against nalidixic acid of 32 to $64 \mathrm{mg} / \mathrm{l}$ and a ciprofloxacin MIC of $1 \mathrm{mg} / 1$. Strains that had a 
high level of resistance ( $>256 \mathrm{mg} / \mathrm{l}$ for nalidixic acid, > $16 \mathrm{mg} / 1$ for ciprofloxacin) accounted for $10.5 \%$ of the $C$ jejuni tested and $21.8 \%$ of the $C$ coli. Resistance to both quinolone drugs tested was more frequent in $C$ coli.

\section{Discussion}

In $C$ coli, $13 \%$ of strains showed resistance to erythromycin, while only $1 \%$ of $C$ jejuni were resistant. This would have a significant bearing on treatment of those infections not caused by $C$ jejuni. Metronidazole has been suggested as a possible alternative treatment in some cases, but this antibiotic is only effective when in an anaerobic atmosphere, this makes testing its efficacy against campylobacter strains difficult, as they will only grow in the presence of low levels of oxygen. $C$ jejuni and $C$ coli were predominantly sensitive to aminoglycosides. Of the 25 strains of $C$ lari tested, 15 were resistant to kanamycin, with one also resistant to neomycin. All $43 C$ jejuni strains which were resistant to kanamycin were also resistant to tetracyclines. This supports the hypothesis that kanamycin resistance is encoded on a plasmid that also mediates tetracycline resistance. ${ }^{14}$

In this study, strains that were resistant to quinolone drugs can be considered in two groups, depending on their MIC. High level resistance predominates, with a further $4.3 \%$ of isolates showing intermediate resistance. $\mathrm{Up}$ to $20 \%$ of patients treated with ciprofloxacin may relapse due to the emergence of resistance with MICs of $32 \mathrm{mg} / \mathrm{l}$ or higher in the infecting strain. ${ }^{7}$ It is therefore wise to avoid treating with ciprofloxacin in those cases where the MIC is already raised. The current British Society for Antimicrobial Chemotherapy (BSAC) recommended breakpoint for low level ciprofloxacin resistance is $1 \mathrm{mg} / \mathrm{l}^{12}$ The closeness of the breakpoint to the sensitive MIC may falsely indicate sensitive strains. The MIC level at which resistance is reported should perhaps be lowered, to encompass all strains showing an increased MIC. Recent sequence analysis of quinolone resistance mutations has shown that a substitution of Thr-86-Ile in the gyrA gene and a Arg-139-Gln substitution in the parC gene of $C$ jejuni is associated with quinolone resistance. ${ }^{15}$ This detailed genetic analysis of resistant mutants will lead to a greater understanding of the evolution and epidemiology of drug resistance in campylobacter.

Reina et al divided nalidixic acid resistant $C$ jejuni strains into two classes ${ }^{6}$ : type 1 , resistant to all quinolones (ciprofloxacin MIC $>4$ $\mathrm{mg} / \mathrm{l}$, nalidixic acid $\mathrm{MIC}>32 \mathrm{mg} / \mathrm{l}$ ), and type 2 , only resistant to acidic quinolones (nalidixic acid MIC > $32 \mathrm{mg} / 1$, ciprofloxacin $\mathrm{MIC}<4$ $\mathrm{mg} / \mathrm{l}$; they do not suggest a mechanism for this resistance. Type 1 predominates in Spain, although figures were only quoted for those strains also resistant to erythromycin (where $8 \%$ of all strains were type 2 and $68 \%$ type 1 ). In the present study, $28 \%$ of nalidixic acid resistant strains fall into type 2, with an MIC against nalidixic acid of between $32 \mathrm{mg} / \mathrm{l}$ and
$64 \mathrm{mg} / \mathrm{l}$ and an MIC against ciprofloxacin of $<4 \mathrm{mg} / \mathrm{l}$.

The link between the use of fluoroquinolones in veterinary medicine and emerging resistance in human isolates should be explored more thoroughly, as it may have serious consequences on treatment of human disease. Concern has been expressed over the rate of ciprofloxacin resistance in Salmonella typhimurium DT104 isolated from humans (14\% in $1996)^{16}$; the rate in Campylobacter spp $(11.7 \%)$ is comparable and there are serious concerns over the apparent rapid increase. ${ }^{7}$ In 1992 , the Expert Group on Animal Feedingstuffs ${ }^{17}$ recommended that antibiotics that could lead to cross resistance to those used in human medicine should not be used as growth promoters, and that their prophylactic use in animals should be reconsidered. This sentiment has been reiterated by the Standing Medical Advisory Committee ${ }^{18}$ subgroup on antimicrobial resistance, which recommend that use of antimicrobial agents in veterinary practice should be guided by the same principles as in human prescribing - that is, that "antimicrobial agents be used only when likely to yield a specific health benefit."

1 Skirrow MB. Campylobacter enteritis: a "new" disease. BMF 1977;ii:9-11.

2 CDSC. Common gastrointestinal infections, England and Wales: laboratory reports weeks 50/97-01/98. Coтmun Dis Rep CDR Wkly 1998;8:14.

3 Frost JA, Oza AN, Thwaites RT, et al. Serotyping scheme for Campylobacter jejuni and Campylobacter coli based on direct agglutination of heat-stable antigens. F Clin Microbiol 1998;36:335-9.

4 Skirrow MB. Diseases due to Campylobacter, Helicobacter and related bacteria. $\mathcal{F}$ Comp Pathol 1994;111:113-49.

5 Reina J, Ros MJ, Serra A. Susceptibilities to 10 antimicrobial agents of 1220 Campylobacter strains isolated from 1987 to 1993 from feces of pediatric patients. Antimicrob Agents Chemother 1994;38:2917-20.

6 Reina J, Ros MJ, Fernandez-Baca V. Resistance to erythromycin in fluoroquinolone-resistant Campylobacter jejuni strains isolated from human faeces [letter]. I Antimicrob Chemother 1995;35:351-2.

7 Piddock LJ. Quinolone resistance and Campylobacter spp. $\mathcal{F}$ Antimicrob Chemother 1995;36:891-8.

8 Bowler IC, Day D. Emerging quinolone resistance in campylobacters. Lancet 1992;340:245.

9 Bowler IC, Connor M, Lessing MP, et al. Quinolone resistance and Campylobacter species [letter]. I Antimicrob Chemother 1996;38:315.

10 McIntyre $M$, Lyons $M$. Resistance to ciprofloxacin in Campylobacter spp [letter; comment]. Lancet 1993;341: 188.

11 Frost JA. Testing for resistance to antimicrobial drugs. In: Chart H, ed. Methods in practical laboratory bacteriology. Boca Raton: CRC Press, 1994:73-82.

12 Working Party of the British Society for Antimicrobial Chemotherapy. A guide to sensitivity testing. F Antimicrob Chemother 1996;38:1103-5.

13 NCCLS. Performance Standards for Antimicrobial Susceptibility Testing. Fifth informational supplement. NCCLS Document M100-S5. Pennsylvania: NCCLS, 1994.

14 Tenover FC, Elvrum PM. Detection of two different kanamycin resistance genes in naturally occurring isolates of Campylobacter jejuni and Campylobacter coli. Antimicrob Agents Chemother 1988;32:1170-3.

15 Gibreel A, Sjogren E, Kaijser B, et al. Rapid emergence of high-level resistance to quinolones in Campylobacter jejuni associated with mutational changes in gyrA and parC. Antimicrob Agents Chemother 1998;42:3276-8.

16 Threlfall EJ, Ward LR, Rowe B. Increasing incidence of resistance to trimethoprim and ciprofloxacin in epidemic Salmonella typhimurium DT104 in England and Wales. Eurosurveillance 1997;2:81-4.

17 Expert Group on Animal Feedingstuffs. Report of the expert group on animal feedingstuffs. London: HMSO, 1992.

18 Standing Advisory Committee SubGroup on Antimicrobial Resistance. The path of least resistance. London: Department of Health, 1998. 